УДК 621.372.8.082.5

А.А. Редюк, ${ }^{1,2}$ О.С. Сидельников ${ }^{1,2}$, Е.А. Аверьянов ${ }^{1}$, М.А. Сорокина ${ }^{3}$ М.П. Федорук ${ }^{1,2}$, С.К. Турицын ${ }^{1,3}$

${ }^{1}$ Новосибирский государственный университет, Новосибирск, Россия

${ }^{2}$ Институт вычислительных технологий СО РАН, Новосибирск, Россия

${ }^{3}$ Институт фоотонных технологий, Университет Астона, Бирмингем, Великобритания

МЕТОД КОМПЕНСАЦИИ НЕЛИНЕЙНЫХ ИСКАЖЕНИЙ СИГНАЛА В ВОЛОКОННЫХ СИСТЕМАХ СВЯЗИ НА ОСНОВЕ ТЕОРИИ ВОЗМУЩЕНИЙ И МАШИННОГО ОБУЧЕНИЯ

В статье исследуется метод компенсации внутриканальных и межканальных нелинейных искажений оптического сигнала для волоконных систем связи со спектральным уплотнением каналов. В его основе лежит дискретная модель, связывающая переданные и принятые символы, построенная на анализе возмущений первого порядка системы уравнений Манакова. Реализация метода осуществляется с помощью решения задачи линейной регрессии с целевой функцией метода наименьших квадратов. На примере трехканальной системы связи протяженностью 2000 км и канальной скоростью передачи 240 Гбит/с показано, что эффективность предлагаемого метода превосходит эффективность линейного восстановления фазы принятого сигнала и находится на уровне метода обратного распространения сигнала с использованием одного шага на пролет.

Ключевые слова: волоконно-оптическая линия связи, система уравнений Манакова, нелинейные искажения сигнала, цифровая обработка сигнала, метод компенсации нелинейных искажений, машинное обучение, теория возмущений. Russian Federation

\author{
A.A. Redyuk ${ }^{1,2}$, O.S. Sidelnikov ${ }^{1,2}$, E.A. Averyanov ${ }^{1}$, \\ M.A. Sorokina ${ }^{3}$, M.P. Fedoruk ${ }^{1,2}$, S.K. Turitsyn ${ }^{1,3}$ \\ ${ }^{1}$ Novosibirsk State University, Novosibirsk, Russian Federation \\ ${ }^{2}$ Institute of Computational Technologies SB RAS, Novosibirsk, Russian Federation \\ ${ }^{3}$ Aston Institute of Photonic Technologies, Aston University, Birmingham, UK

\section{PERTURBATIVE MACHINE LEARNING TECHNIQUE FOR NONLINEAR IMPAIRMENTS COMPENSATION IN FIBER OPTIC COMMUNICATION SYSTEMS}

In the paper anapproach of intra- and inter-channel nonlinear impairments compensation for optical signal in wave-division multiplexingfiber communication systems is investigated. The approach is 
based on a discrete model connecting the transmitted and received symbols, built on the first-order perturbation analysis of the Manakov equations. The method works by linear regression with the objective function of least squares method. It is shown on the example of a three-channel communication system with a length of $2000 \mathrm{~km}$ and a channel transmission rate of $240 \mathrm{Gbit} / \mathrm{s}$ that efficiency of the proposed method exceeds efficiency of the phase shift equalization of the received signal and is at the level of the digital back propagation using one step per span.

Keywords: fiber optic communication systems, Manakov equations, nonlinear signal distortions, digital signal processing, nonlinear distortion compensation method, machine learning, perturbation theory.

\section{Введение}

Нелинейные искажения оптического сигнала, возникающие при его распространении по волоконно-оптическим линиям связи (ВОЛС), остаются среди основных ограничивающих факторов, препятствующих дальнейшему увеличению дальности и скорости передачи информации по ВОЛС [1]. В особенности это касается современных когерентных систем связи со спектральным уплотнением каналов (WDM систем), подверженных помимо внутриканальных также и межканальным нелинейным взаимодействиям. Для уменьшения влияния данного эффекта на качество передачи информации было предложено и исследовано несколько классов методов подавления и компенсации нелинейных искажений. Среди них можно выделить следующие: методы обратного распространения сигнала (Digital Back Propagation, DBP) [2], основанные на численном решении нелинейного уравнения Шредингера в обратном направлении; методы, основанные на использовании функционального ряда Вольтера [3]; методы предыскажения сигнала и посткомпенсации нелинейных эффектов (Perturbation-based Post Equalization, PPE) [4, 5], основанные на использовании элементов теории возмущений. Однако практическое применение этих методов сопряжено с рядом трудностей, главным образом связанных с их высокой вычислительной сложностью, а следовательно, невозможностью их аппаратной реализации и использования в режиме реального времени. Данная проблема потенциально может быть решена с помощью привлечения обширного арсенала методов машинного обучения. Данное направление бурно развивается и находит большое число применений в области нелинейной фотоники в последние несколько лет $[6,7,8]$.

В работе [9] авторы рассматривают модель внутриканальных и межканальных нелинейных взаимодействий на примере распространения оптического сигнала, состоящего из двухспектральных каналов с использованием двух поляризаций в каждом канале. Для компенса- 
ции нелинейных искажений переданного сигнала с помощью предлагаемой модели на основе теории возмущений необходимо вычислить тысячи коэффициентов возмущений, выраженных многомерными осциллирующими интегралами, число которых только растет с увеличением числа каналов. В работе [10] авторами предлагается вместо численного интегрирования для нахождения коэффициентов использовать методы регрессионного анализа. Показано, что такой подход демонстрирует высокую эффективность метода при значительно уменьшенной вычислительной трудоемкости для оптических систем связи с пространственным уплотнением каналов по модам сигнала. В данной работе мы обобщаем и применяем этот метод для компенсации внутриканальных и межканальных нелинейных эффектов в системах связи со спектральным уплотнением каналов, используя информацию с соседних по частоте каналов, чтобы восстановить изначальный сигнал.

\section{Метод компенсации нелинейных искажений сигнала}

Анализ возмущений первого порядка уравнений Манакова, используемых для моделирования распространения по волоконному световоду оптического сигнала с двумя возбужденными поляризациями, позволяет перейти от непрерывной модели на комплексную огибающую сигнала к дискретной модели, связывающей символы на передатчике и символы на приемнике [9]:

$$
X_{k}=x_{k}+\Delta x_{k}, \quad Y_{k}=y_{k}+\Delta y_{k},
$$

где $x_{k}$ и $y_{k}$ - комплексные амплитуды импульсов во временном слоте $k$ на передатчике для $x$ - и $y$-поляризации соответственно, аналогично $X_{k}$ и $Y_{k}$ - комплексные амплитуды импульсов на приемнике. Величины $\Delta x_{k}$ и $\Delta y_{k}-$ некоторая оценка накопленных за время распространения сигнала по волокну нелинейных искажений, учитывающая кубическую нелинейность среды и основанная на взаимодействии троек импульсов с двух поляризаций. В случае учета одного спектрального канала данная оценка дается следующим выражением [5]:

$$
\Delta x_{k}=\sum_{m, n} C_{m n}\left(x_{k+m} x_{k+n} \bar{x}_{k+m+n}+x_{k+m} y_{k+n} \bar{y}_{k+m+n}\right),
$$

где верхнее подчеркивание обозначает комплексное сопряжение. Аналогичные выражения для $y$-поляризации получаются заменой символов 
$x$ на $y$ и наоборот. Коэффициенты $C_{m n}$ из (2) - комплексные коэффициенты возмущений, зависящие от характеристик волокна, сигнала и протяженности линии. Аналитические выражения для $C_{m n}$ в виде многомерных интегралов можно найти в работах $[9,10,11]$, однако в силу их громоздкости в данной статье они не приводятся.

В случае учета нескольких спектральных каналов, распространяющихся одновременно, выражение (2) можно обобщить и записать в следующем виде:

$$
\begin{gathered}
\Delta x_{k}^{s}=\sum_{m, n} C_{m n}\left(x_{k+m}^{s} x_{k+n}^{s} \bar{x}_{k+m+n}^{s}+x_{k+m}^{s} y_{k+n}^{s} \bar{y}_{k+m+n}^{s}\right)+ \\
+\sum_{c \neq 0} \sum_{m, n} D_{m n}^{c}\left(2 x_{k+m}^{s} x_{k+n}^{s+c} \bar{x}_{k+m+n}^{s+c}+x_{k+m}^{s} y_{k+n}^{s+c} \bar{y}_{k+m+n}^{s+c}+y_{k+m}^{s} x_{k+n}^{s+c} \bar{y}_{k+m+n}^{s+c}\right),
\end{gathered}
$$

где $s$ - номер рассматриваемого канала.

В частных случаях можно получить компактные приближенные аналитические выражения для коэффициентов $C_{m n}$ [12], однако в случаях, интересных с практической точки зрения, вычисление коэффициентов требует ресурсозатратного численного интегрирования. Основная идея предлагаемого в статье метода состоит в поиске коэффициентов возмущений и $D_{m n}^{c}$ с помощью методов машинного обучения на заранее известном тренировочном наборе передаваемых и принятых символов. После этапа обучения найденные коэффициенты применяются уже на тестовом наборе для компенсации накопленных нелинейных эффектов и восстановления переданных символов по правилу:

$$
x_{k}^{s}=X_{k}^{s}-\Delta x_{k}^{s}, \quad y_{k}^{s}=Y_{k}^{s}-\Delta y_{k}^{s} .
$$

Заметим, что для вычисления «нелинейных» поправок $\Delta x_{k}^{s}$ и $\Delta y_{k}^{s}$ с помощью выражения (3) необходимо не только знание коэффициентов возмущений $C_{m n}$ и $D_{m n}^{c}$, но и знание самих передаваемых символов $x_{k}$ и $y_{k}$ со всех каналов. В рассматриваемом методе в качестве их приближения используются принятые символы $X_{k}$ и $Y_{k}$ после восстановления фазы линейным компенсатором.

\section{Модель волоконной системы связи}

Для оценки эффективности предлагаемого метода посткомпенсации нелинейных искажений, основанного на возмущениях первого по- 
рядка (РРЕ), были выполнены численные эксперименты передачи данных по ВОЛС, принципиальная схема которой представлена на рис. 1. Моделировалось распространение оптического сигнала, состоящего из трех спектральных каналов с двумя возбужденными поляризациями. В каждом канале сигнал задавался в виде последовательности импульсов в форме «корня приподнятого косинуса» (Root-Raised Cosine, RRC) с коэффициентом сглаживания 0,01 с форматом модуляции 16QAM и символьной скоростью 32 Гбод. Таким образом, полная скорость передачи данных в одном канале составляла 256 Гбит/с, полезная скорость передачи - 240 Гбит/с. Несущая длина волны центрального канала составляла 1550 нм, межканальное расстояние - 37,5 ГГц. Для компенсации оптических потерь в линии моделировались точечные эрбиевые усилители после каждого из 20 пролетов длиной 100 км с коэффициентом шума 4,5 дБ. На приемнике после демультиплексирования сигнала выполнялись идеальная компенсация накопленной хроматической дисперсии, взятие отсчетов с согласованным фильтром, цифровая компенсация нелинейных искажений и анализ битовых ошибок. Количество передаваемых символов для тренировочной и тестовой последовательности составляло $2^{16}$ символов на каждую поляризацию, что соответствует $2^{19}$ передаваемых битов в одном канале. Коэффициент битовой ошибки всегда измерялся для центрального канала.

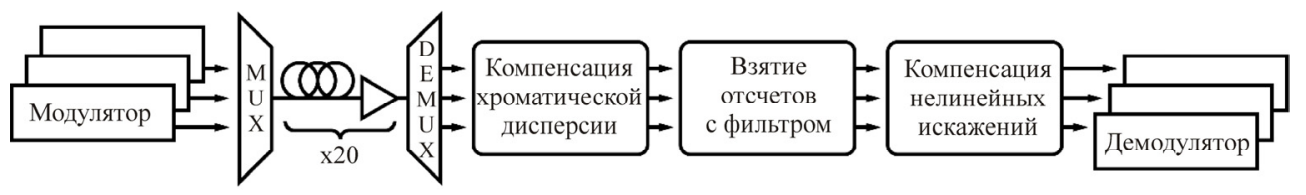

Рис. 1. Принципиальная схема исследуемой ВОЛС: MUX-мультиплексор сигнала, DEMUX - демультиплексор

Распространение оптического сигнала по волоконному световоду описывалось системой уравнений Манакова:

$$
\frac{\partial A_{x, y}}{\partial z}=\left(-\frac{\alpha}{2}-i \frac{\beta_{2}}{2} \frac{\partial^{2}}{\partial t^{2}}+i \gamma \frac{8}{9}\left[\left|A_{x}\right|^{2}+\left|A_{y}\right|^{2}\right]\right) A_{x, y},
$$

где $A_{x, y}(z, t)$ - комплексная огибающая электрического поля для $x$ - и $y$-поляризаций, коэффициент затухания $\alpha=0,2$ дБ/км, параметр дисперсии второго порядка $D=17$ пс/нм/км, коэффициент нелинейности 
$\gamma=1,4$ 1/Вт/км. Для данных уравнений ставилась начально-краевая задача с периодическими граничными условиями. Начальные условия задавались в виде:

$$
\begin{aligned}
& A_{x}(z=0, t)=\sqrt{P_{0} / 2} \sum_{s} \sum_{k} x_{k}^{s} f(t-k T) e^{i \Omega s t}, \\
& A_{y}(z=0, t)=\sqrt{P_{0} / 2} \sum_{s} \sum_{k} y_{k}^{s} f(t-k T) e^{i \Omega s t},
\end{aligned}
$$

где $P_{0}$ - вводимая в волокно канальная мощность сигнала, $f(t)$ - форма импульса, $T$ - длительность символьного интервала, $\Omega$ - межканальное расстояние. Задача решалась численно с помощью симметричной схемы метода расщепления по физическим процессам с использованием преобразования Фурье второго порядка точности по эволюционной переменной с 16 отсчетами на символ.

\section{Результаты численных экспериментов и обсуждение}

При проведении численных экспериментов всегда выполнялось два независимых расчета с различными реализациями случайных последовательностей символов на передатчике и величин, моделирующих шумы усилителей: один расчет для получения тренировочной последовательности символов (для вычисления коэффициентов возмущений) и один расчет для получения тестовой последовательности символов (для тестирования предлагаемого метода).

Для нахождения коэффициентов возмущений $C_{m n}$ и $D_{m n}^{c}$ формировалась система линейных алгебраических уравнений на основе тренировочной последовательности и ставилась задача линейной регрессии. Для ее решения использовались функционалы, соответствующие методу наименьших квадратов, гребневой регрессии, методу LASSO (Least Absolute Shrinkageand Selection Operator). Их оптимизация осуществлялась методом среднего стохастического градиента [13], что позволило выполнять вычисления в комплексной арифметике. Поиски коэффициентов с разными целевыми функционалами показали близкие результаты, и основным отличием их применения является сложность вычисления, поэтому мы остановились в своей работе на методе наименьших квадратов как на самом простом и наиболее изученном методе. 
По причине дисперсионного уширения импульсов при распространении по волокну и влияния импульсов из соседних временных интервалов на рассматриваемый импульс эффективность метода РРЕ зависит от параметра $R$, который определяет диапазон суммирования в (3) по правилу $|m|+|n| \leq R$. С увеличением $R$ эффективность метода монотонно растет, пока не достигает порогового значения, которое сохраняется при дальнейшем росте $R$. На рис. 2 представлен характерный вид матриц $\left\{C_{m n}\right\}$ и $\left\{D_{m n}^{c}\right\}$ для ограничений $|m|+|n| \leq 35$ и $|m|+|n| \leq 25$ соответственно. Как видно, матрицы имеют выделяющиеся линии вдоль осей $m=0$ и $n=0$, что свидетельствует о том, что наибольшее влияние на импульс в своем временном слоте имеет он сам в сравнении с импульсами в других временных слотах. При удалении от центра $m=n=0$ абсолютная величина коэффициентов экспоненциально уменьшается вплоть до полного обнуления, что свидетельствует о конечном дисперсионном наплывании соседних импульсов друг на друга.
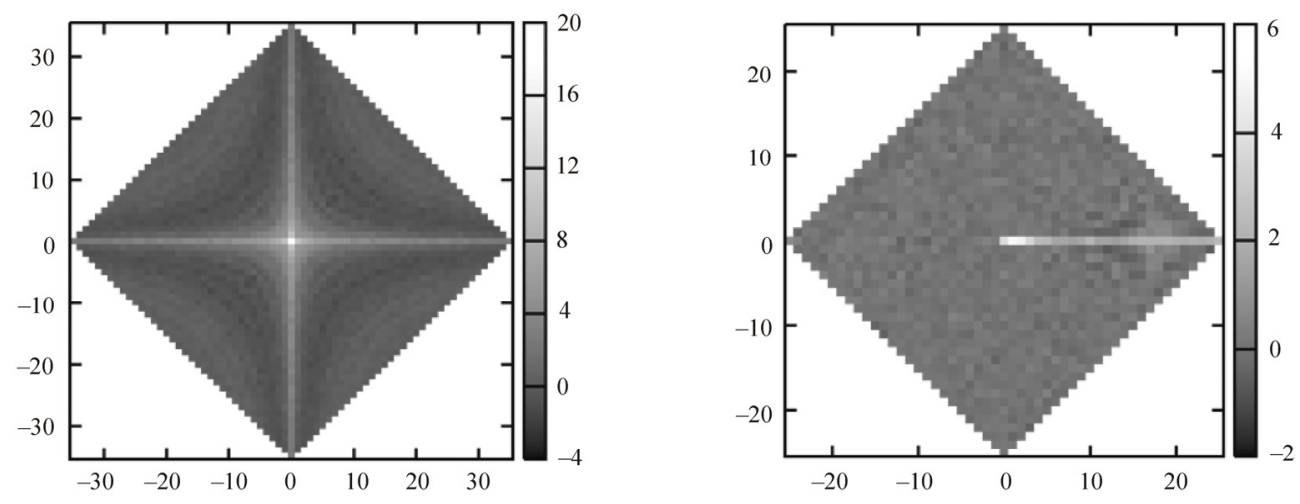

Рис. 2. Мнимая часть модельных коэффициентов возмущений. Слева матрица $\left\{C_{m n}\right\}$, справа $\left\{D_{m n}^{1}\right\}$

Для оценки эффективности предлагаемого метода РРЕ он сравнивался с другими методами компенсации нелинейных искажений: линейным компенсатором, который только восстанавливает фазу принятого сигнала (Phase-Shift Equalization, PSE), и методом обратного распространения сигнала с использованием одного (DBP1) и двух (DBP2) вычислительных шагов по эволюционной переменной на один пролет. На рис. 3 представлена зависимость коэффициента битовой ошибки (BER) от вводимой в волокно канальной мощности для четырех рас- 
сматриваемых методов компенсации нелинейных искажений. Из графика видно, что метод PPE, обгоняя PSE, работает на одном уровне c DBP1 и достигает наименьшего показателя коэффициента ошибки $4,7 \cdot 10^{-3}$ при мощности сигнала 0,5 дБм. Следует отметить, что с увеличением количества шагов на пролет для метода обратного распространения сигнала коэффициент битовых ошибок уменьшается, но в то же время пропорционально растет вычислительная сложность данного метода.

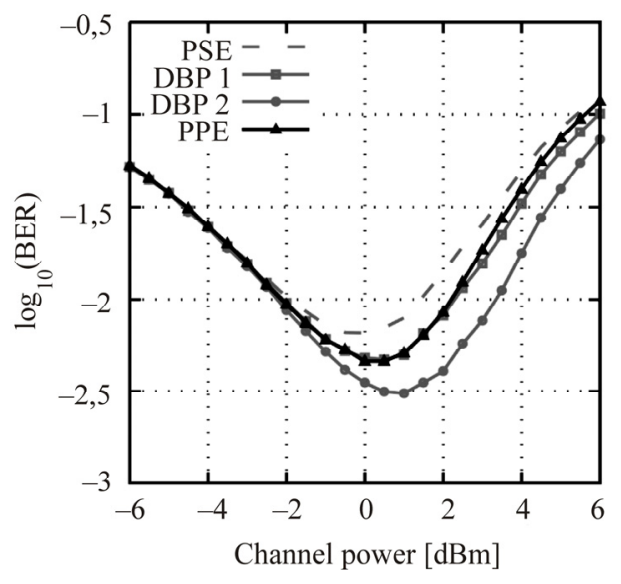

Рис. 3. Зависимость коэффициента битовой ошибки от канальной мощности вводимого сигнала для различных методов компенсации нелинейных искажений

На рис. 4 показаны сигнальные созвездия принятого сигнала после компенсации нелинейности методом PSEи PPE для вводимой в волновод канальной мощности 0,5 дБм.
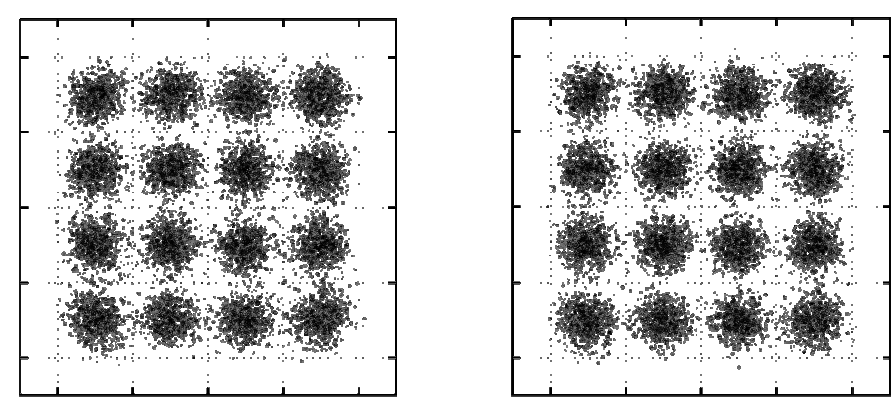

Рис. 4. Сигнальные созвездия после применения методов PSE (слева) и РPE (справа) 
На рис. 5 представлена зависимость коэффициента битовой ошибки от длины распространения сигнала при оптимальной мощности сигнала для каждой длины. На графике изображены линии, соответствующие тем же методам компенсации искажений, что и на рис. 3. Следует отметить, что при передаче сигналов на расстояние порядка 1000-1400 км эффективность предлагаемого метода РРЕ оказывается выше метода обрат-

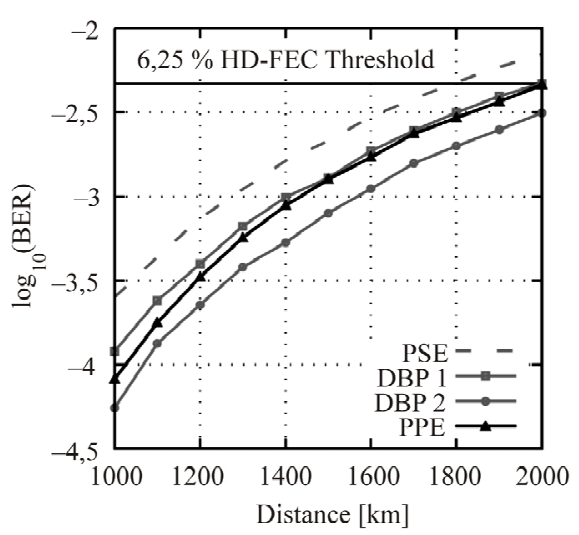

Рис. 5. Зависимость коэффициента битовой ошибки от длины распространения для различных методов компенсации нелинейных искажений ного распространения с использованием одного шага на пролет DBP1. Видно, что в случае применения техники коррекции ошибок (FEC) из работы [14] с фиксированным пороговым значением BER, при котором еще возможно его использование, метод РРЕ позволяет увеличить расстояние передачи на 200 км с 1800 до 2000 км при сохранении того же уровня ошибок по сравнению с методом PSE.

Важно отметить, что хотя метод DBP1 и демонстрирует результат, близкий к методу РРЕ, его вычислительная сложность остается высокой, что мешает его практическому применению. В то же время РРЕ лишен такого недостатка, что делает его подходящим для компенсации нелинейных искажений в современных когерентных ВОЛС. Кроме того, метод РРЕ допускает периодическое переобучение через определенные интервалы времени, что позволяет использовать его в линиях связи с динамически изменяемыми параметрами, в то время как метод обратного распространения может применяться только в статических системах.

\section{Заключение}

В работе исследован метод компенсации внутриканальных и межканальных нелинейных эффектов в системах связи со спектральным уплотнением каналов, основанный на теории возмущении. Было проведено численное сравнение эффективности рассматриваемого ме- 
тода с линейным компенсатором PSE и методом обратного распространения сигнала с использованием одного и двух шагов на пролет. Полученные результаты показывают, что метод РРЕ обеспечивает лучшее качество передачи данных, чем PSE, и работает на одном уровне с DBP1. Кроме того, при применении кода, исправляющего ошибки, предлагаемый метод позволяет увеличить расстояние передачи на 200 км при сохранении того же уровня ошибок по сравнению с линейным восстановлением фазы принятого сигнала.

Исследование выполнено при финансовой поддержке РФФИ и правительства Новосибирской области в рамках научного проекта № 17-42-543379.

\section{Список литературы}

1. Overcoming Kerr-induced capacity limit in optical fiber transmission / E. Temprana, E. Myslivets, B.P.-P. Kuo, L. Liu, V. Ataie1, N. Alic, S. Radic // Science. - 2015. - Vol. 348(6242). - P. 1445-1448.

2. Ip E. Nonlinear compensation using backpropagation for polarization-multiplexed transmission // Journal of Lightwave Technology. - 2010. Vol. 28(6). - P. 939-951.

3. Liu L., Li L., Huang Y. Intrachannel nonlinearity compensation by inverse Volterra series transfer function // Journal of Lightwave Technology. - 2012. - Vol. 30(3). - P. 310-316.

4. Robust and efficient receiver-side compensation method for intrachannel nonlinear effects / T. Oyama, H. Nakashima, S. Oda, T. Yamauchi, Z. Tao, T. Hoshida, J.C. Rasmussen // Proceedings of Optical Fiber Communication Conference. - San Francisco. - 2014. - Paper Tu3A.3

5. Multiplier-free intrachannel nonlinearity compensating algorithm operating at symbol rate / Z. Tao, L. Dou, W. Yan, L. Li, T. Hoshida, J.C. Rasmussen // Journal of Lightwave Technology. - 2011. - Vol. 29(17). P. 2570-2576.

6. Machine learning techniques in optical communication / D. Zibar, M. Piels, R. Jones, C.G. Schäeffer // Journal of Lightwave Technology. 2016. - Vol. 34(6). - P. 1442-1452.

7. Sidelnikov O.S., Redyuk A.A., Sygletos S. Dynamic neural network-based methods for compensation of nonlinear effects in multimode 
communication lines // Quantum Electronics. - 2017. - Vol. 47(12). P. 1147-1149.

8. Support vector machines for error Correction in Optical Data Transmission / A. Metaxas, A. Redyuk, Y. Sun, A. Shafarenko, N. Davey, R. Adams // Lecture Notes in Computer Science. - 2013. - Vol. 7824. P. 438-445.

9. Inter-channel nonlinear interference noise in WDM systems: modeling and mitigation / R. Dar, M. Feder, A. Mecozzi, M. Shtaif // Journal of Lightwave Technology. - 2015. - Vol. 33(5). - P. 1044-1053.

10. Sorokina M., Sygletos S., Turitsyn S. Sparse identification for nonlinear optical communication systems: SINO method // Optics Express. 2016. - Vol. 24. - P. 30433-30443.

11. Mecozzi A. A unified theory of intrachannel nonlinearity in pseudolinear transmission // Impact of Nonlinearities on Fiber Optic Communications / ed. S. Kumar. - New York, NY: Springer New York, 2011. P. 253-291.

12. Ghazisaeidi A., Essiambre R.-J. Calculation of coefficients of perturbative nonlinear pre-compensation for Nyquist pulses // Proceedings of European Conference on Optical Communication. - Cannes, 2014. - P. 1-3.

13. Schmidt M., Le Roux N., Bach F. Minimizing finite sums with the stochastic average gradient // Mathematical Programming. - 2017. Vol. 162(1). - P. 83-112.

14. Zhang L.M., Kschischang F.R. Staircase codes with 6 to $33 \%$ overhead // Journal of Lightwave Technology. - 2014. - Vol. 32(10). P. 1999-2001.

\section{References}

1. Temprana E., Myslivets E., Kuo B.P.-P., Liu L., Ataie1V., Alic N., Radic S. Overcoming Kerr-induced capacity limit in optical fiber transmission. Science, 2015, vol. 348(6242), pp. 1445-1448.

2. Ip E. Nonlinear compensation using backpropagation for polarization-multiplexed transmission. Journal of Lightwave Technology, 2010, vol. 28(6), pp. 939-951.

3. Liu L., Li L., Huang Y. Intrachannel nonlinearity compensation by inverse Volterra series transfer function. Journal of Lightwave Technology, 2012, vol. 30(3), pp. 310-316. 
4. Oyama T., Nakashima H., Oda S., Yamauchi T., Tao Z., Hoshida T., Rasmussen J.C. Robust and efficient receiver-side compensation method for intra-channel nonlinear effects. Proceedings of Optical Fiber Communication Conference. San Francisco, 2014, paper Tu3A.3.

5. Tao Z., Dou L., Yan W., Li L., Hoshida T., Rasmussen J.C. Multiplier-free intrachannel nonlinearity compensating algorithm operating at symbol rate. Journal of Lightwave Technology, 2011, vol. 29(17), pp. 2570-2576.

6. Zibar D., Piels M., Jones R., Schäeffer C.G. Machine learning techniques in optical communication. Journal of Lightwave Technology, 2016, vol. 34(6), pp. 1442-1452.

7. Sidelnikov O.S., Redyuk A.A., Sygletos S. Dynamic neural network-based methods for compensation of nonlinear effects in multimode communication lines. Quantum Electronics, 2017, vol. 47(12), pp. 1147-1149.

8. Metaxas A., Redyuk A., Sun Y., Shafarenko A., Davey N., Adams R. Support vector machines for error Correction in Optical Data Transmission. Lecture Notes in Computer Science, 2013, vol. 7824, pp. 438-445.

9. Dar R., Feder M., Mecozzi A., Shtaif M. Inter-channel nonlinear interference noise in WDM systems: modeling and mitigation. Journal of Lightwave Technology, 2015, vol. 33(5), pp. 1044-1053.

10. Sorokina M., Sygletos S., Turitsyn S. Sparse identification for nonlinear optical communication systems: SINO method. Optics Express, 2016, vol. 24, pp. 30433-30443.

11. Mecozzi A. A unified theory of intrachannel nonlinearity in pseudolinear transmission. Impact of Nonlinearities on Fiber Optic Communications. Ed. S. Kumar. New York, NY, Springer New York, 2011, pp. 253-291.

12. Ghazisaeidi A., Essiambre R.-J. Calculation of coefficients of perturbative nonlinear pre-compensation for Nyquist pulses. Proceedings of European Conference on Optical Communication. Cannes, 2014, pp. 1-3.

13. Schmidt M., Le Roux N., Bach F. Minimizing finite sums with the stochastic average gradient. Mathematical Programming, 2017, vol. 162(1), pp. 83-112.

14. Zhang L.M., Kschischang F.R. Staircase codes with 6 to $33 \%$ overhead. Journal of Lightwave Technology, 2014, vol. 32(10), pp. 19992001.

Получено 26.09.2018 